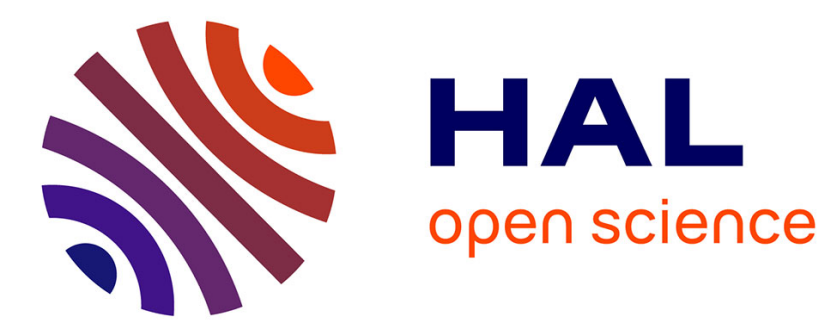

\title{
Estimating inbreeding depression for growth and reproductive traits using pedigree and genomic methods in Argentinean Brangus cattle
}

Natalia S Forneris, Carolina A Garcia-Baccino, Rodolfo J C Cantet, Zulma G. Vitezica

\section{- To cite this version:}

Natalia S Forneris, Carolina A Garcia-Baccino, Rodolfo J C Cantet, Zulma G. Vitezica. Estimating inbreeding depression for growth and reproductive traits using pedigree and genomic methods in Argentinean Brangus cattle. Journal of Animal Science, 2021, 99, 10.1093/jas/skab289 . hal-03482733

\section{HAL Id: hal-03482733 https://hal.science/hal-03482733}

Submitted on 16 Dec 2021

HAL is a multi-disciplinary open access archive for the deposit and dissemination of scientific research documents, whether they are published or not. The documents may come from teaching and research institutions in France or abroad, or from public or private research centers.
L'archive ouverte pluridisciplinaire HAL, est destinée au dépôt et à la diffusion de documents scientifiques de niveau recherche, publiés ou non, émanant des établissements d'enseignement et de recherche français ou étrangers, des laboratoires publics ou privés. 
ANIMAL GENETICS AND GENOMICS

Estimating inbreeding depression for growth and reproductive traits using pedigree and genomic methods in Argentinean Brangus cattle

\author{
Natalia S. Forneris, ${ }^{\dagger,+1}$ Carolina A. Garcia-Baccino, ${ }^{\dagger}$ Rodolfo J. C. Cantet, ${ }^{\dagger, \ddagger}$ and \\ Zulma G. Vitezicall
}

†Departamento de Producción Animal, Facultad de Agronomía, Universidad de Buenos Aires, C1417DSQ Buenos Aires, Argentina, ${ }^{\ddagger}$ CONICET-Universidad de Buenos Aires, Instituto de Investigaciones en Producción Animal (INPA), C1427CWO Buenos Aires, Argentina, "INP/INRAE, GenPhySE, 31326 Castanet-Tolosan, France

${ }^{1}$ Corresponding author: forneris@agro.uba.ar

ORCiD numbers: 0000-0002-4052-5333 (N. S. Forneris); 0000-0002-1465-5708 (C. A. Garcia-Baccino).

\begin{abstract}
Inbreeding depression reduces the mean phenotypic value of important traits in livestock populations. The goal of this work was to estimate the level of inbreeding and inbreeding depression for growth and reproductive traits in Argentinean Brangus cattle, in order to obtain a diagnosis and monitor breed management. Data comprised 359,257 (from which 1,990 were genotyped for 40,678 single nucleotide polymorphisms [SNPs]) animals with phenotypic records for at least one of three growth traits: birth weight (BW), weaning weight (WW), and finishing weight (FW). For scrotal circumference (SC), 52,399 phenotypic records (of which 256 had genotype) were available. There were 530,938 animals in pedigree. Three methods to estimate inbreeding coefficients were used. Pedigree-based inbreeding coefficients were estimated accounting for missing parents. Inbreeding coefficients combining genotyped and nongenotyped animal information were also computed from matrix $\mathrm{H}$ of the single-step approach. Genomic inbreeding coefficients were estimated using homozygous segments obtained from a Hidden Markov model (HMM) approach. Inbreeding depression was estimated from the regression of the phenotype on inbreeding coefficients in a multiple-trait mixed model framework, either for the whole dataset or for the dataset of genotyped animals. All traits were unfavorably affected by inbreeding depression. A $10 \%$ increase in pedigree-based or combined inbreeding would result in a reduction of 0.34 to $0.39 \mathrm{~kg}$ in BW, 2.77 to $3.28 \mathrm{~kg}$ in WW, and $0.23 \mathrm{~cm}$ in SC. For FW, a $10 \%$ increase in pedigree-based, genomic, or combined inbreeding would result in a decrease of 8.05 to $11.57 \mathrm{~kg}$. Genomic inbreeding based on the HMM was able to capture inbreeding depression, even in such a compressed genotyped dataset.
\end{abstract}

Key words: Argentinean Brangus beef cattle, growth and reproductive traits, inbreeding depression, pedigree-based and genomic inbreeding coefficients

\section{Introduction}

An important topic in animal breeding today is the state of genetic variability. Offspring from related mates yields a higher probability of sharing homologous alleles identical by descent across the whole genome, also called inbreeding (Malécot, 1948). Selection in livestock populations results not only in remarkable genetic gain but also in an accumulation of inbreeding. The most important observed consequence of inbreeding is a reduction of 


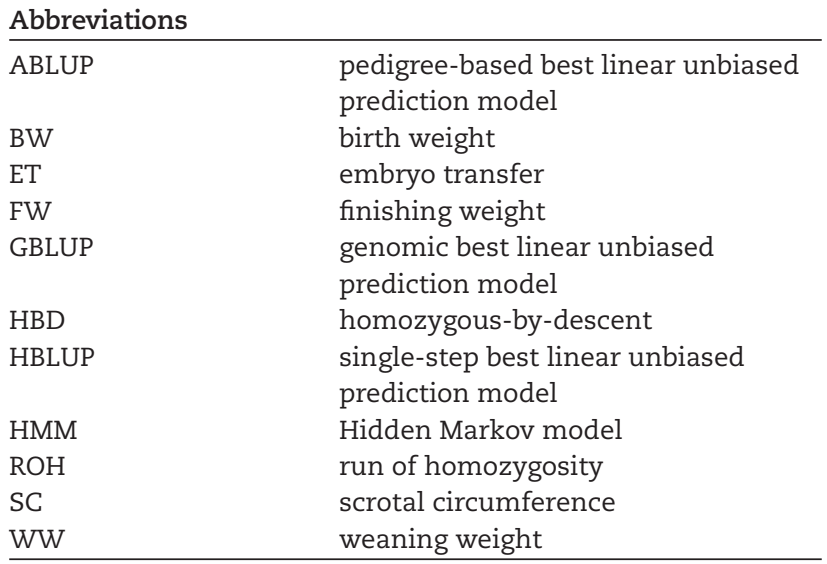

the mean phenotypic value of a trait. This phenomenon is known as inbreeding depression (Falconer and Mackay, 1996). Since the 1960s (Brinks et al., 1965; see also the review by Burrow, 1993), until more recently (Carrillo and Siewerdt, 2010; Pereira et al., 2016; Sumreddee et al., 2018; Garcia-Baccino et al., 2020), there is an extensive literature on the effects of inbreeding depression on several traits of beef cattle. Prior to the genomic era, the inbreeding coefficient of an individual has been computed from pedigree information, as the kinship coefficient of its parents. The latter is defined as the probability that the two alleles which the individual receives at a given locus are identical by descent (Jacquard, 1974, p. 108). However, computation of the inbreeding coefficient from pedigree assumes an infinitesimal model with unrelated loci. Genomic approaches are expected to be more accurate because marker-based inbreeding coefficients reflect that inheritance (Mendelian sampling and linkage) is passed on in chromosomes (e.g., Baes et al., 2019). Different molecular methods to estimate inbreeding coefficients exist, ignoring (single-SNP based) or using information from contiguous markers. The runs of homozygosity (ROH; e.g., McQuillan et al., 2008 ) is an approach that provides for an alternative measure of inbreeding, because it attempts to estimate the joint occurrence of inbreeding in neighboring markers. However, the definition of $\mathrm{ROH}$ is rule based and somewhat arbitrary as there is no clear association of the statistics to a proper distribution that is framed on population or quantitative genetics (Peripolli et al., 2017). Alternatively, a Hidden Markov model (HMM) approach was proposed to identify homozygous segments to estimate individual inbreeding and to infer the age of inbreeding (Druet and Gautier, 2017).

Argentinean Brangus is a composite breed that combines traits of Angus (carcass quality and fertility) and Brahman (heat and disease tolerance) cattle. In most of the countries that currently breed Brangus cattle, the founder breed composition has been fixed at five-eighths Angus and three-eighths Zebu (mainly Brahman). However, the Argentinean Brangus cattle were developed differently from U.S. Brangus cattle in the sense that the breed composition is variable, due to the need to provide an answer to harsh subtropical environments containing ticks, parasites, low-quality forages, and hot, humid weather (Cantet, 2013). On average, the Argentinean Brangus displays one-third Zebu genome and two-thirds Angus genome (Goszczynski et al., 2018). Thus, animals with different fractions of Zebuine genes coexist in a range going from one-eighth to five-eighths depending on the geographical region. The larger fraction of Zebu was intended to promote even better adaptation to warm and humid environments. Since its creation in 1978, the Argentinean Brangus Breeders Association (AAB, Asociación Argentina de Brangus; https://brangus.com.ar) has kept an open registry policy to maintain high levels of genetic variability (Ron Garrido et al., 2008). The AAB carries out a genetic evaluation program routinely since 1999 that considers growth and reproductive and carcass quality traits. In beef cattle, characters, such as birth weight (BW), weaning weight (WW), finishing weight (FW), and scrotal circumference (SC), are subject to intensive selection. The reproductive trait SC is associated with the fertility of the bull and sexual precocity of his daughters The goal of this research was to estimate the level of inbreeding and inbreeding depression for growth (BW, WW, and FW) and the reproductive trait SC in Argentinean Brangus cattle. Pedigree-based, genomic, and combined approaches were used on the whole dataset and on a subset of animals, respectively.

\section{Material and Methods}

\section{Animals and records}

Animal Care and Use Committee approval was not obtained for this study because the data were taken from an existing database. We employed two datasets for all of the analyses. The first one, hereafter referred to as the whole dataset (ALL), consists of the 359,257 (181,359 males and 177,898 females) animals enrolled in the genetic evaluation program of the AAB, with records for at least one of the three growth traits (BW, WW, and FW). A total of 52,399 animals in ALL were recorded for SC. There were 530,938 animals in the pedigree that were born between 1950 and 2019. The other set, hereafter referred to as the genotyped dataset (GEN), consisted of a subset of 1,990 animals (731 males and 1,259 females), with genotype and phenotype information for growth traits, born between 2008 and 2019. For SC, only 256 animals had genotype. Figure 1 displays the distribution of the genotyped animals across year of birth. The genotyped animals were mostly (81\%) young candidates (Figure 1; (71\% of them born after 2017) with no genotyped progeny in the dataset.

Table 1 presents a summary of the data for the growth (BW, WW, and FW) and reproductive traits (SC). WW was measured on average at 6.5 mo of age, whereas FW and SC were measured at $18 \mathrm{mo}$ of age, on average. Phenotypes fulfilled quality requirements of the AAB's genetic evaluation program.

Animals were genotyped for 47,843 SNPs with the GeneSeek-Genomic-Profiler Bovine 50K version 2 panel (Neogen Corporation, Lincoln, NE). Quality control was performed using PREGSF90 (Aguilar et al., 2014). Animals with a genotyping call rate below $90 \%$ were removed. Markers with a call rate lower than $90 \%$, with a minor allele frequency lower than $5 \%$, and with Mendelian conflicts were removed. After quality control, 40,678 autosomal SNPs and 1,990 animals were used for the analysis.

\section{Pedigree and genomic inbreeding coefficients}

Pedigree-based inbreeding coefficients $\left(F_{\text {Ped }}\right)$ were calculated using the method of VanRaden (1992) that calculates inbreeding accounting for missing parents. In this approach, animals with missing parents were assumed to have inbreeding coefficients equal to the mean of the inbreeding coefficients for animals with known parents born during the same year. This approach is suitable for ALL (whole dataset), as in Brangus almost $40 \%$ of the animals have either parent unknown due to the open policy 


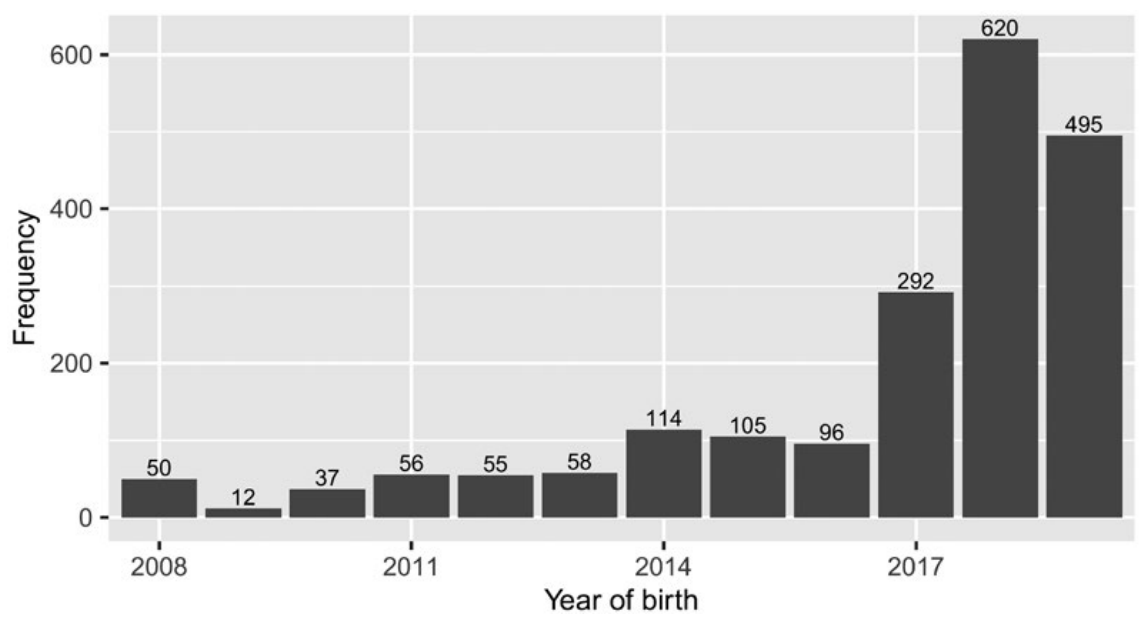

Figure 1. Year of birth counts on the Brangus genotyped set $(n=1,990)$.

Table 1. Number of records, average, standard deviation (SD), and number of contemporary groups (CG) of the evaluated growth and reproductive traits

\begin{tabular}{|c|c|c|c|c|c|c|c|c|}
\hline \multirow[b]{2}{*}{ Trait $^{1}$} & \multirow[b]{2}{*}{ No. of records } & \multicolumn{3}{|c|}{$\mathrm{ALL}^{2}$} & \multicolumn{4}{|c|}{$\mathrm{GEN}^{2}$} \\
\hline & & Mean & $\mathrm{SD}$ & CG & No. of records & Mean & SD & CG \\
\hline BW, kg & 314,437 & 34.01 & 4.80 & 3,596 & 1,964 & 35.59 & 4.81 & 62 \\
\hline WW, kg & 238,561 & 187.34 & 43.50 & 4,275 & 1,668 & 225.50 & 50.82 & 53 \\
\hline FW, kg & 119,673 & 385.70 & 99.21 & 3,074 & 981 & 481.16 & 100.79 & 68 \\
\hline $\mathrm{SC}, \mathrm{cm}$ & 52,399 & 33.63 & 3.69 & 1,078 & & & & \\
\hline
\end{tabular}

${ }^{1} \mathrm{BW}$, birth weight; FW, finishing weight; SC, scrotal circumference; WW, weaning weight.

${ }^{2} \mathrm{ALL}$, whole dataset; GEN, genotyped dataset.

of registration and the use of multiple-sire natural mating (Ron Garrido et al., 2008). The algorithm proposed by Aguilar and Misztal (2008) implemented in the program INBUPGF90 was used to estimate inbreeding coefficients in ALL.

Genomic inbreeding coefficients were computed in GEN using the HMM approach proposed by Druet and Gautier (2017), which estimates the proportion of the genome contained in locally inbred segments. Their approach allows refining the genomic partitioning of inbreeding into stretches of homozygous-by-descent (HBD) segments from possibly different ancestral origins. This approach relies on the fact that, normally in livestock populations, multiple ancestors from different generations contribute to the inbreeding of an individual. The model estimates inbreeding relative to multiple age-related classes, being each inbreeding distribution associated with a different time in the past. The HMM consists of $K$ hidden classes corresponding to 1 non-HBD class and $K-1$ different HBD classes, each characterized by its own mixing coefficient. The latter coefficient is defined as the proportion of HBD segments of class $c$ in the genome $(c \in(1, K-1))$, and rate $G_{c}$, which is approximately equal to twice the age (in generations) to the common ancestors associated with class c. Classes with lower ages correspond to longer HBD segments from more recent common ancestors. The model uses marker allele frequencies, genetic distances, and the sequences of observed genotypes, while accounting for genotyping error. The HMM-based approach is implemented in the program ZooRoH.f90 and is freely available at https://github. com/tdruet/ZooRoH.
According to the available marker density, we ran a model consisting of a set with nine classes $(K=9)$ : eight HBD classes with predefined rates (interpreted as ages for the inbreeding classes) $G_{c}$ ranging from 2 to 256 (with $G_{c}=2^{c}$ for each class $c \in$ $(1,8)$, for instance, $G_{8}=256$ ) and one non-HBD class. The program outputs the genome-wide inbreeding $F_{\mathrm{ZooRoH}}=F_{\mathrm{G}}=\sum_{c=1}^{8} F_{\mathrm{G}}^{(c)}$, where $F_{\mathrm{G}}^{(c)}$ is the inbreeding associated with each HBD class $c$. The choice of a model with predefined age-related classes instead of a model in which ZooRoH estimates them was motivated by the possibility of getting clues on the actual (true) ages of inbreeding and comparing all the animals according to the same age-based partitioning of inbreeding.

Alternatively, in order to have a comparable measure of inbreeding for both genotyped and ungenotyped animals and to extract the maximum information from the data, we estimated inbreeding in ALL as $F_{H_{i}}=H_{i i}-1$ (i.e., through the diagonal of the single-step covariance matrix $\mathrm{H}$ ) using the method of Legarra et al. (2020) with a single metafounder and fixed allele frequencies in $\mathrm{G}$ equal to 0.5 . For genotyped animals, this results in the diagonal of $\mathrm{H}$ being proportional to homozygosity (Garcia-Baccino et al., 2017), whereas for nongenotyped animals, this results in a genomically enhanced prediction of homozygosity from the expression: $H_{11}^{(\gamma)}=A_{11}^{(\gamma)}+A_{12}^{(\gamma)} A_{22}^{(\gamma)-1}\left(G_{05}-A_{22}^{(\gamma)}\right) A_{22}^{(\gamma)-1} A_{21}^{(\gamma)}$.

Pearson's correlation coefficient was calculated among the pedigree-based $\left(F_{\mathrm{Ped}}\right)$, ZooRoHs $\left(F_{\mathrm{ZooRoH}}\right)$, and matrix $\mathrm{H}$ inbreeding $\left(F_{H}\right)$ for animals in the corresponding datasets. Regressions of 
$F_{\text {ZooRoH }}$ or $F_{H}$ on $F_{\text {Ped }}$ and of $F_{H}$ on $F_{\text {ZooRoH }}$ were used as a measure of the quality of the estimation.

\section{Statistical models}

Inbreeding depression was estimated in both datasets: ALL (whole dataset) and GEN (only genotyped animals). Phenotypes were analyzed using three animal models: a pedigree-based (ABLUP), a single-step (HBLUP), and a genomic (GBLUP) models. Multi-trait ABLUP, HBLUP, and GBLUP models for growth traits were fitted to ALL and GEN. Single-trait ABLUP and HBLUP model $\mathrm{SC}$ in ALL. GBLUP was not run for SC due to the reduced number of genotyped animals with records for this trait.

For ALL, the ABLUP (or HBLUP) model for BW, WW, and FW was equal to:

$\left[\begin{array}{l}y^{\mathrm{BW}} \\ y^{\mathrm{WW}} \\ y^{\mathrm{FW}}\end{array}\right]=\mathbf{X}_{1}\left[\begin{array}{c}\beta^{\mathrm{BW}} \\ \beta^{\mathrm{WW}} \\ \beta^{\mathrm{FW}}\end{array}\right]+\mathbf{X}_{2}\left(\left[\begin{array}{l}b^{\mathrm{BW}} \\ b^{\mathrm{WW}} \\ b^{\mathrm{FW}}\end{array}\right] \otimes F\right)+Z\left[\begin{array}{l}u^{\mathrm{BW}} \\ u^{\mathrm{WW}} \\ u^{\mathrm{FW}}\end{array}\right]+Z_{m}\left[\begin{array}{c}0 \\ m a t \\ 0\end{array}\right]+\left[\begin{array}{c}e^{\mathrm{BW}} \\ e^{\mathrm{WW}} \\ e^{\mathrm{FW}}\end{array}\right]$

where $y^{t}$ is the vector of observed phenotypic values for trait $t$ (with $t$ being one of $\mathrm{BW}, \mathrm{WW}$, or $\mathrm{FW}), \mathrm{u}^{\mathrm{t}}$ stands for the vector of direct breeding values, mat stands for the vector of maternal breeding values (only for $\mathrm{WW}$ ), and $e^{t}$ stands for the error term. $\mathbf{X}_{1}$ and $\mathbf{X}_{2}$ are design matrices relating the phenotype to the fixed effects; $Z$ and $Z_{m}$ are incidence matrices relating the phenotype with the direct and maternal breeding values, respectively. The vector of fixed effects for each trait, $\beta^{t}=\left[\mu^{t} \text { hys }{ }^{t} S^{t} A D^{t} a^{t}\right]^{\prime}$, included the mean $\left(\mu^{t}\right)$, the contemporary group (hyst) -defined for animals within the same herd-year-season (for BW and WW) or within the same herd-year-season-sex (for FW), the sex of the animal ( $S^{\text {t; }} 2$ levels; only for BW and WW), and the age class of the dam of the animal ( $A D^{t} ; 6$ levels; only for BW and WW). The six classes for $A D^{t}$ were 2, 3, 4, 5 to 8, 9, or more years of age and an additional class involving embryo transfer (ET) animals. The regression coefficient $\left(a^{t}\right)$ of the observed phenotype on the covariate age of the animal in days since birth, at which trait $t$ was actually measured, was included in $\boldsymbol{\beta}^{\mathrm{t}}$ just for WW and FW. The term $b^{t} F$ models the inbreeding depression, where $b^{t}$ is the inbreeding depression parameter per unit of inbreeding, and the covariate $F$ is the vector of individual inbreeding coefficients, either $F_{\text {Ped }}$ for ABLUP or $F_{H}$ for HBLUP. Normal distributions were assumed for random effects, with $[$ umat $] \sim N\left(\mathbf{0}, G_{0} \otimes \mathbf{A}\right)$ for ABLUP or $N\left(0, G_{0} \otimes H\right)$ for HBLUP, and $e \sim N\left(0, R_{0} \otimes I\right)$, where $A$ is the pedigree-based additive relationship matrix and $H$ is the single-step matrix (Legarra et al., 2009; Christensen and Lund, 2010). Variance component values in $G_{0}$ and $R_{0}$ were assumed known and obtained from the national genetic evaluation (values in Supplementary Material).

For SC, the ABLUP (or HBLUP) model fitted to ALL was defined as:

$$
\boldsymbol{y}^{S C}=\mathbf{X} \boldsymbol{\beta}^{S C}+b^{S C} \mathbf{F}+Z u^{S C}+e^{S C}
$$

where $y^{S C}$ is the vector of observed phenotypic values for SC and the vector of fixed effects, $\beta^{\mathrm{SC}}=\left[\begin{array}{ll}\mu^{\mathrm{SC}} \text { hys } & \mathrm{SC}^{\mathrm{SC}}\end{array}\right]^{\prime}$, included the mean $\left(\mu^{\mathrm{SC}}\right)$, the contemporary group (hys $\left.{ }^{\mathrm{SC}}\right)$, and the regression coefficent $\left(a^{S C}\right)$ of the observed phenotype on the covariate age of the animal in days since birth, at which SC was actually measured. The remaining terms, that is, $b^{S C} F, u^{S C}, e^{S C}, X$, and $Z$ were defined as in the multi-trait ABLUP model. It was assumed that $u^{S C} \sim N\left(0, A \sigma_{A}^{2}\right)$ for ABLUP or $N\left(0, H \sigma_{A}^{2}\right)$ for HBLUP, and that
$e^{\mathrm{SC}} \sim \mathrm{N}\left(0, I \sigma_{e}^{2}\right)$, where $\sigma_{\AA}^{2}$ and $\sigma_{e}^{2}$ are the additive genetic and residual variance, respectively.

For GEN, the GBLUP model used for BW, WW, and FW was:

$$
\left[\begin{array}{l}
y^{\mathrm{BW}} \\
y^{\mathrm{WW}} \\
y^{\mathrm{FW}}
\end{array}\right]=\mathbf{X}_{1}\left[\begin{array}{l}
\boldsymbol{\beta}^{\mathrm{BW}} \\
\boldsymbol{\beta}^{\mathrm{WW}} \\
\boldsymbol{\beta}^{\mathrm{FW}}
\end{array}\right]+\mathbf{X}_{2}\left(\left[\begin{array}{l}
b^{\mathrm{BW}} \\
b^{\mathrm{WW}} \\
b^{\mathrm{FW}}
\end{array}\right] \otimes \boldsymbol{F}_{\mathrm{ZooRoH}}\right)+Z\left[\begin{array}{l}
u^{\mathrm{BW}} \\
u^{\mathrm{WW}} \\
u^{\mathrm{FW}}
\end{array}\right]+\left[\begin{array}{l}
e^{\mathrm{BW}} \\
e^{\mathrm{WW}} \\
e^{\mathrm{FW}}
\end{array}\right]
$$

where the vector of fixed effects for trait $t$ (with $t$ being one of BW, WW, or FW), $\boldsymbol{\beta}^{\mathrm{t}}=\left[\mu^{\mathrm{t}} h^{\mathrm{t}} \text { yob }{ }^{\mathrm{t}} S^{\mathrm{t}} A D^{\mathrm{t}} a^{\mathrm{t}}\right]^{\prime}$ included $h_{j}^{t}$. The latter parameter was defined for animals within the same herd (for BW and WW) or within the same herd and sex (for FW). The effect yobt $(12$ levels) is the year of birth. Both were treated as cross-classified effects. The remaining effects, that is, $S^{t}, A D^{t}$, and $a^{t}$, were defined as in the ABLUP model, except the inbreeding coefficients, $F_{\text {ZooRoH }}$, that were those estimated using ZooRoH. Maternal effects were completely confounded in GEN and were not included when analyzing this set. Among all dams of animals in GEN (1,271 individuals), 999 (79\% of dams) had only one calf, leading to an average of 1.52 offspring per cow. Moreover, 55\% of the genotyped animals (i.e., in GEN) were born as a result of ET, without any record of the recipient dam. This value is far bigger than the $9.7 \%$ ET animals observed in ALL. In this model, $\mathbf{u} \sim \mathrm{N}\left(\mathbf{0}, \boldsymbol{G}_{0} \otimes \mathbf{G}\right)$ (values in Supplementary Material). The additive genomic relationship matrix $G$ was calculated according to VanRaden (2008) as follows:

$$
\mathbf{G}=\frac{\mathbf{M M}^{\prime}}{2 \sum_{k=1}^{m} p_{k}\left(1-p_{k}\right)}
$$

where $\mathbf{M}$ is a matrix with dimensions of number of animals $(n)$ by number of SNP $(m)$, with elements equal to $\left(2-2 p_{k}\right)$, $\left(1-2 p_{k}\right)$, and $\left(-2 p_{k}\right)$, for genotypes $\mathrm{AA}, \mathrm{A} a$, and $a a$, respectively; $p_{k}$ is the frequency for allele $A$ of SNP $k$.

Additionally, in order to measure globally the strength of evidence of inbreeding depression, the signals from the three growth traits were pooled to build a Hotelling's t-squared statistic with a single P value, as follows. Let $\hat{\boldsymbol{b}}=\hat{\boldsymbol{\beta}}_{[: i \mathrm{i}+2]}=\left(\hat{b}^{\mathrm{BW}}, \hat{b}^{\mathrm{WW}}, \hat{b}^{\mathrm{FW}}\right)$ be a subset of $\hat{\boldsymbol{\beta}}$ starting at position $i$ with the estimated inbreeding depression coefficients for each trait obtained from either the multi-trait ABLUP, HBLUP, or GBLUP evaluation. Following Chen et al. (2017) and Legarra et al. (2018), the statistic is a quadratic form $\left.x=\hat{b}^{\prime}\left(\boldsymbol{\Sigma}_{[i: i+2,} \quad i: i+2\right]\right)^{-1} \hat{\boldsymbol{b}}$, where $\boldsymbol{\Sigma}=\operatorname{var}(\hat{\boldsymbol{\beta}})=C^{\boldsymbol{\beta} \boldsymbol{\beta}}$ is the posterior covariance matrix of the estimators. The submatrix $\mathrm{C}_{[i: i+2, i: i+2]}^{\boldsymbol{\beta} \boldsymbol{\beta}}$ can be obtained by running BLUPF90test with the "store_pev_pec" option. Under multivariate normality, $x$ follows a chi-square distribution with 3 degrees of freedom.

Programs of the BLUPF90 family (Misztal et al., 2002) were used for the analyses and are available at http://nce.ads.uga.edu/wiki/ doku.php. The GBLUP analysis was run as is described at http:// nce.ads.uga.edu/wiki/doku.php?id=how_to_run_pure_gblup.

\section{Results}

The mean of the inbreeding coefficients across individuals $\left(F_{\text {Ped }}\right)$ was equal to 0.039 with an SD of 0.014 , a result that is similar to the one reported by Paim et al. (2020) for American Brangus. Pedigree-based inbreeding increased over the years, reaching an average value of $4 \%$ in 2019 (Figure 2). Only $0.07 \%$ of the evaluated animals were non-inbred, and around $96.87 \%$ 
presented values of the inbreeding coefficient up to $6.25 \%$, $2.57 \%$ between $6.25 \%$ and $12.50 \%, 0.34 \%$ between $12.50 \%$ and $18.75 \%, 0.02 \%$ between $18.75 \%$ and $25 \%$, and $0.13 \%$ above $25 \%$. The mean of the inbreeding coefficients calculated from $\mathrm{H}$ matrix $\left(F_{H}\right)$ was equal to 0.131 with an SD of 0.015 . Only $0.03 \%$ of the evaluated animals presented values of combined inbreeding up to $6.25 \%, 16.98 \%$ between $6.25 \%$ and $12.50 \%$, $81.98 \%$ between $12.50 \%$ and $18.75 \%, 0.81 \%$ between $18.75 \%$ and $25 \%$, and $0.2 \%$ above $25 \%$. Inbreeding calculated from $\mathrm{H}$ matrix using pedigree and genotypes with metafounder relationships reduced the proportion of animals (from $96.87 \%$ to $0.03 \%$ ) with inbreeding coefficient less than $6.25 \%$ compared with pedigree-based method. The correlation between $F_{H}$ and $F_{\text {Ped }}$ was 0.79 , and the regression slope was equal to 0.9 for the whole dataset.

For animals in the genotyped set (GEN), the mean (SD) of pedigree-based inbreeding coefficients $\left(F_{P e d}\right)$ was $0.047(0.019)$,

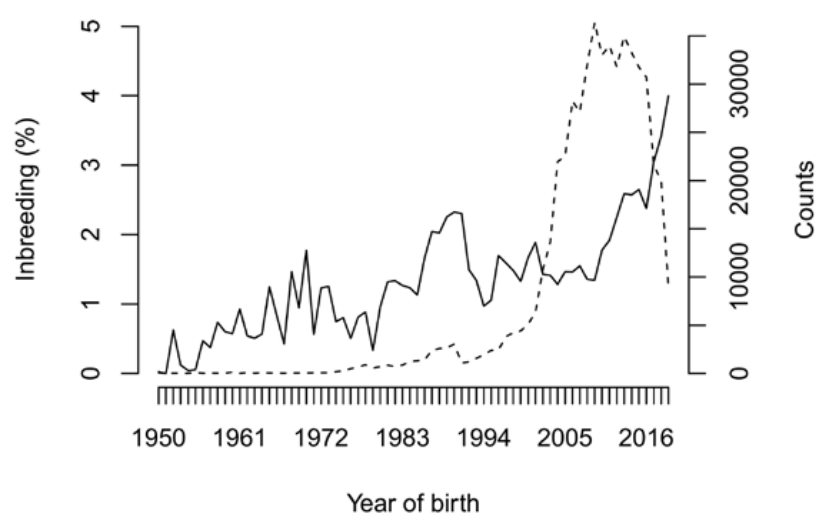

Figure 2. Year of birth counts (dashed line) and average pedigree-based inbreeding coefficient, \% (black line), for animals in the national evaluation between years 1950 and 2019. with a minimum of 0.027 and a maximum of 0.279 . The mean of genomic inbreeding coefficients $\left(F_{\mathrm{ZooRoH}}\right)$ was equal to 0.127 with an SD of 0.029 , with a minimum of 0.008 and a maximum of 0.334. For the animals in GEN, the correlation between $F_{\text {ZooRoH }}$ and $F_{\text {Ped }}$ was 0.49 (with a slope of 0.75 ), while between $F_{\text {ZooRoH }}$ and $F_{H}$ was 0.95 with a slope approximately equal to 1 .

Besides providing a global estimator of inbreeding for each individual, the model implemented in ZooRoH was informative of the partition of individual inbreeding. When plotting the distributions of the estimated inbreeding contributions (proportion of the individual genomes) assigned to each of the eight predefined HBD classes (Figure 3), these were concentrated in the HBD class with predefined age-based rates equal to 16 (G16), 32 (G32), and, in a lesser extent, to the oldest HBD class (G256).

This becomes more noticeable when observing the estimated mean contribution of each predefined HBD class (averaged over all the animals; Figure 4a) and the corresponding mean cumulative genomic inbreeding (Figure $4 b$ ). The peaks of inbreeding in the HBD classes correspond to a period of reduced $\mathrm{N}_{e}$ or to an immediate neighboring one.

Assuming a generation interval of approximately $5 \mathrm{yr}$ for beef cattle, the peak around classes G16 and G32 (between 8 and 16 generations to most recent ancestor; Figures 3 and $4 a$ ) is consistent with the bottlenecks associated with the relatively recent breed formation in Argentina from a small number of founders from each parental breed (mainly, Angus and Brahman and in a lesser extent Nellore).

The peak of inbreeding in the older HBD class G256 (Figures 3 and 4a) may be associated with ancient inbreeding, which captures ancient demographic history (past $N_{e}$ and resulting LD) and presents less variation among individuals. ZooRoH automatically assigns such inbreeding to the eldest HBD classes. This class may be also capturing some of the segments that actually belong to more recent classes (when, e.g., genotyping errors break them) together with a small proportion of those with an older time to most recent ancestor, because the performance

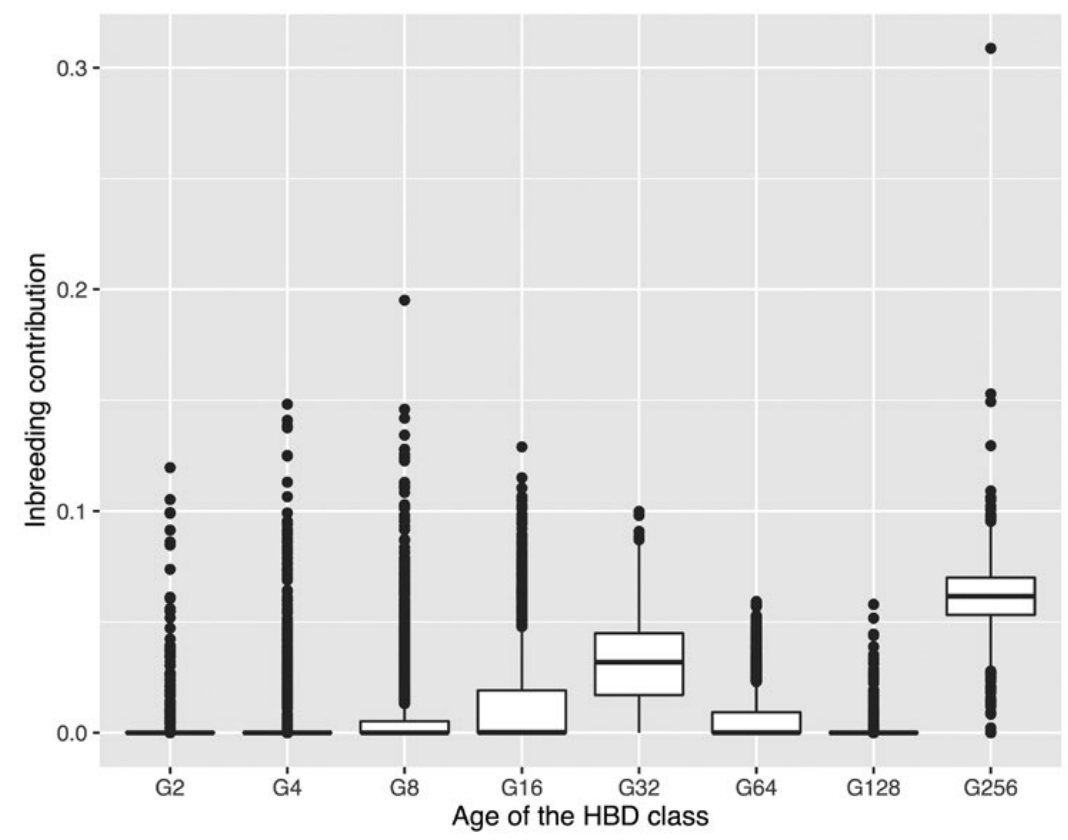

Figure 3. Distributions of the estimated inbreeding contributions (proportion of the individual genomes) $F_{\mathrm{G}}^{(c)}$ assigned to each of the eight predefined homozygous-bydescent (HBD) classes (over the 1,990 genotyped individuals). 
a

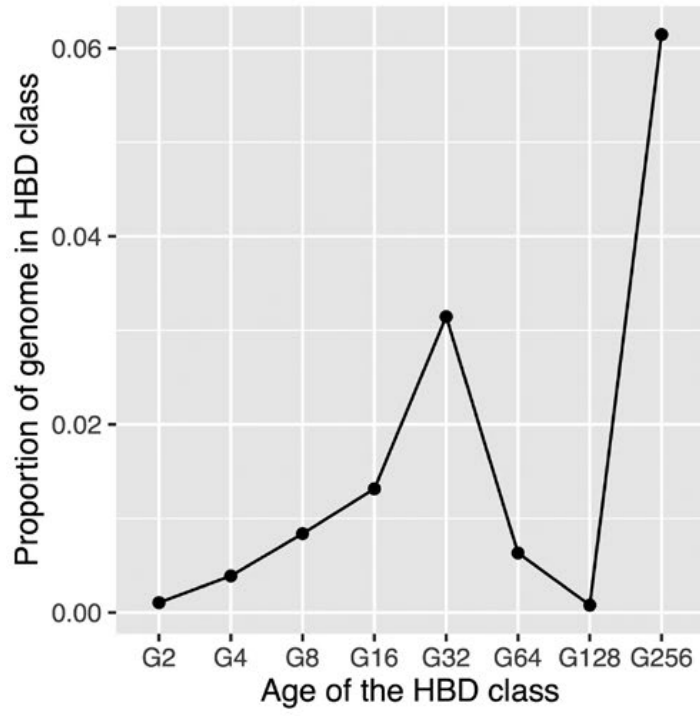

b

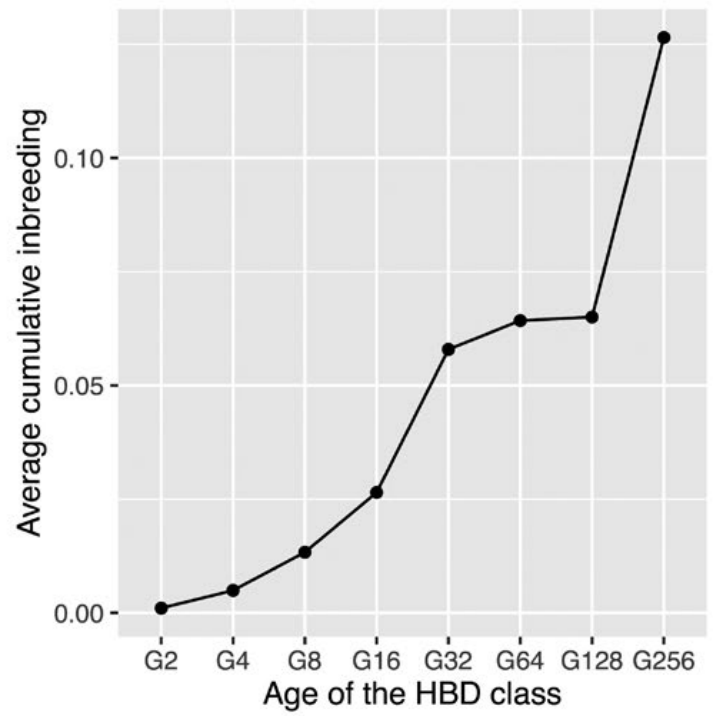

Figure 4. Average estimated proportions of inbreeding contribution (a) and corresponding average cumulative inbreeding (b) of a set of eight predefined homozygousby-descent (HBD) classes for the Brangus population.

of ZooRoH in correctly assigning HBD segments declines as the timing of the bottleneck is older (Druet and Gautier, 2017).

Inbreeding depression for each growth trait was estimated using multi-trait ABLUP and HBLUP in the whole dataset (ALL) and GBLUP in the genotyped set (GEN). For SC, inbreeding depression was estimated using single-trait ABLUP and HBLUP in ALL. Inbreeding depression estimates (Table 2) are expressed as the change in phenotypic mean per $10 \%$ increase in inbreeding. For ALL, both ABLUP and HBLUP yielded similar inbreeding depression coefficients that were statistically significant ( $P$-values $<0.05$ ) for the four traits. For BW and WW, the values of inbreeding depression obtained with GBLUP for the genotyped set were very close to those obtained from ABLUP and HBLUP in ALL. Our estimates of inbreeding depression obtained with GBLUP were negative for BW and WW but nonsignificantly different from zero. For FW, the inbreeding depression estimated with GBLUP in the genotyped set was $44 \%$ and $13 \%$ higher compared with ABLUP and HBLUP, respectively, and the $95 \%$ confidence estimation interval excluded the zero value. For this trait, a decrease of either $8.05,10.27$, or $11.49 \mathrm{~kg}$ is expected every $10 \%$ increase in pedigreebased, combined, and marker-based inbreeding, respectively.

From the posterior covariance matrix of estimates for the three growth traits, the Hotelling's t-squared statistic was computed. The results indicated a global significant effect for either ABLUP (P-value: $4.45 \times 10^{-13}$ ) or HBLUP estimates (P-value: $\left.1.46 \times 10^{-11}\right)$ and a nonsignificant global effect ( $P$-value: 0.102$)$ for GBLUP estimates in our dataset.

\section{Discussion}

The Argentinean Brangus breed has an average value of pedigree-based inbreeding equal to $4 \%$. Genomic inbreeding was equal to $13 \%$ using a combined pedigree and genomic approach $\left(F_{H}\right)$ for the whole set of animals (ALL) and $12.7 \%$ using a markerbased approach $\left(F_{\mathrm{ZooRoH}}\right)$ for the genotyped animals (GEN). A high correlation (0.95) was obtained between $F_{\text {ZooRoH }}$ and $F_{H}$. As expected, pedigree-based inbreeding $\left(F_{\text {Ped }}\right)$ was moderately correlated (0.49) with marker-based inbreeding ( $\left.F_{\text {ZooRoH }}\right)$ for animals in GEN; realized inbreeding is expected to deviate from
Table 2. Inbreeding depression for growth and reproductive traits estimated by multi-trait pedigree-based (ABLUP), single-step (HBLUP), and genomic (GBLUP) BLUP

\begin{tabular}{cccc}
\hline Trait ${ }^{2}$ & $\begin{array}{c}b^{1} \text { with } \\
\text { ABLUP-F }\end{array}$ & $\begin{array}{c}b^{1} \text { with } \\
\text { GBLUP- } F_{\text {ZooRoH }}\end{array}$ \\
\hline BW, kg & $-0.341(0.076)^{*}$ & $-0.394(0.082)^{*}$ & $-0.410(0.327)$ \\
WW, kg & $-2.772(0.557)^{*}$ & $-3.283(0.604)^{*}$ & $-2.890(2.256)$ \\
FW, kg & $-8.051(1.354)^{*}$ & $-10.271(1.419)^{*}$ & $-11.572(5.210)^{*}$ \\
SC, cm & $-0.232(0.099)^{*}$ & $-0.237(0.101)^{*}$ & \\
\hline
\end{tabular}

${ }^{1}$ Inbreeding depression estimates are expressed as the change in phenotypic mean, per 10\% increase in inbreeding (SE are in parenthesis).

${ }^{2} \mathrm{BW}$, birth weight; FW, finishing weight; SC, scrotal circumference; WW, weaning weight.

${ }^{*} \mathrm{P}<0.05$.

the pedigree-based expectation due to Mendelian sampling and linkage (Hill and Weir, 2011). Yet, on average, genomic inbreeding was higher than pedigree-based inbreeding $\left(F_{\text {Ped }}\right)$. Probably, this result is due to the pedigree information being often incomplete to some extent or to the genotyping strategy. With respect to the pedigree incompleteness, grade dams are an important source of founders in Argentinean Brangus, whose calves with records are routinely registered in the national evaluation. Oftentimes, most of these cows are paternal half-sibs that result from natural matings, which in turn may yield an underestimated $F_{P e d}$ . On the other hand, genotyped animals are probably individuals with the highest estimated breeding values and strongly related among them (from the same bull-sire families). Indeed, the phenotypic mean for WW and FW was significantly higher (P-value $<0.05)$ for GEN than for ALL. Also, the average pedigreebased relationship between animals in GEN was equal to 0.03 , being $14 \%$ of these pairwise relationships above 0.0625 (halfcousins) and with a maximum of 0.71 .

Breeding of Brangus cattle began in Argentina in the 1950s and nowadays is the second breed in number of registrations after Angus. The expansion took place mainly in Northeastern and Central regions of the country, especially after the creation 
of the Argentinean Brangus Breeders Association in 1978. The effective population size of the Brangus breed in Argentina has been estimated in 274 under random mating, and 125 under selection, using pedigree records of animals born between 1959 and 2005 (Ron Garrido et al., 2008). Also, the same authors, with 21,662 Brangus calves born between 2001 and 2005, reported an effective number of founders of 765 and an effective number of ancestors of 387. These values are higher than those reported in other beef cattle breeds mainly due to the breed's open policy of registration. This open policy, which eventually leads to incomplete parent identification (almost $40 \%$ of the animals have either parent unknown), may result in overestimated $N_{e}$ (and underestimated inbreeding values) from pedigree records. The increase in average inbreeding $(\Delta \mathrm{F})$ obtained by linear regression of the average annual inbreeding coefficient with PED $\left(F_{\text {Ped }}\right)$ over the studied years was equal to $0.039 \%$ per year, which is lower than the $2 \%$ acceptable in commercial breeds according to Food and Agriculture Organization's (FAO's) guidelines for in vivo conservation of animal genetic resources (FAO, 2013). Based on this value, the resulting effective population size, using a generation interval of 5.1 (Ron Garrido et al., 2008) and the formula $N_{e}=1 / 2 \Delta F$ (Falconer and Mackay, 1996), was equal to 250 . This is smaller to the value of 274 obtained by Ron Garrido et al. (2008) with records up to 2005 and agrees with the fact that inbreeding has increased in the last years.

Overall, estimates of inbreeding depression were in agreement with those reported in the literature (with and without genomic information) for growth traits in beef cattle. For BW, the estimates reported are variable: some studies suggested no inbreeding depression (Burrow, 1998; Davis and Simmen, 2010), while others presented values going from -0.12 to $-3.80 \mathrm{~kg}$ per $10 \%$ increase in inbreeding (Swiger et al., 1961; Burrow, 1993; Sumreddee et al., 2018, 2020; Garcia-Baccino et al., 2020). In our case, the values obtained were within this range $(-0.341,-0.394$, and $-0.410 \mathrm{~kg}$ for ABLUP, HBLUP, and GBLUP, respectively). For WW, estimates of inbreeding depression were also within the range of values reported in literature, going from -2.12 to $-10.20 \mathrm{~kg}$ per $10 \%$ increase in inbreeding (Burrow, 1993, 1998; Falcão et al., 2001; Santana et al., 2010; Sumreddee et al., 2018; Garcia-Baccino et al., 2020). Burrow (1998) reported a decrease of $14.90 \mathrm{~kg}$ per $10 \%$ increase in inbreeding for FW (weight at 18 months). This value is higher than those obtained in the current research (estimated with either ABLUP, HBLUP, or GBLUP). Some differences between populations are expected given that estimates of inbreeding depression from subtropical or tropical breeds are population specific. Finally, for SC, our estimates of inbreeding depression $(-0.23 \mathrm{~cm})$ were within the range of values reported using pedigree, going from -0.03 to $-0.7 \mathrm{~cm}$ per $10 \%$ increase in inbreeding (Burrow, 1993, 1998; Pereira et al., 2016). To our knowledge, there are no estimates of inbreeding depression for SC in beef cattle using genomic methods.

Inbreeding depression is believed to be caused by the impact from recessive detrimental mutations or, alternatively, by losing the advantage from alleles with heterozygous superiority (Charlesworth and Willis, 2009). Partial dominance seems to account for a large proportion of the inbreeding depression (Curik et al., 2014; Leroy, 2014). Our results agree with the dominant idea that both productive (growth; as BW, WW, and FW) and reproductive traits such as SC are negatively affected by inbreeding depression (Charlesworth and Willis, 2009) in beef cattle (Brinks et al., 1965; Burrow, 1993). Both with pedigreebased (ABLUP) and combined (HBLUP) approaches, inbreeding depression was detected for all traits. The trait BW displays positive genetic correlations with WW and FW close to 0.31 and 0.35 , respectively. The latter traits are negatively affected by inbreeding depression. Therefore, a reduction in BW by inbreeding depression may induce a reduction in WW and thus an unfavorable inbreeding depression is attained for FW.

Using genomic (GBLUP) information, only FW showed inbreeding depression. One main difference between our ABLUP (or HBLUP) and GBLUP was the number of phenotypes. While ABLUP (or HBLUP) used all the available phenotypes (ALL), GBLUP used only records from genotyped animals that were $0.7 \%$ of ALL. Model specification also differed between ABLUP (or HBLUP) and GBLUP in contemporary group (CG) and maternal effects. In ABLUP (or HBLUP), CG classes were defined based on herd, year, and season. In GBLUP, the reduced dataset (genotyped animals) forced us to eliminate the "season" effect, and "herd" and "year" were not treated together. In addition, maternal effects were not included in the GBLUP analysis because they were completely confounded. These differences may have affected the SE of estimates and can explain why inbreeding depression was only confirmed for FW in GBLUP. To better support this idea, we ran an extra ABLUP analysis (results not shown) using only the phenotypes of the genotyped animals and only FW showed inbreeding depression.

Recently, Villanueva et al. (2021) showed that the diagonal elements of matrix $G$, as are computed in our study (VanRaden, 2008, method 1), cannot be interpreted as inbreeding coefficients. First, inbreeding coefficients were computed using an HMM approach (ZooRoH) and not from $G$. Second, inbreeding depression models the mean of an individual, whereas the selfrelationship models its variance. The diagonal of $G$ (or of $A$ ) matrix contains the self-relationship of an individual, which is the a priori variance of its breeding value with respect to some base population. For instance, fixing the diagonal of $G$ matrix to 1 would result in over- or under-dispersion of estimated breeding values of corresponding animals, but it does not affect estimate of inbreeding depression, which is just a covariate. Thus, GBLUP (or ABLUP) needs to have a properly defined relationship matrix that is unrelated to the estimate of inbreeding depression.

Inbreeding is increasing in the Argentinean Brangus population and it has a detrimental effect on growth and reproductive traits. These findings lead us to stress on the importance of balancing genetic improvement and maintaining genetic variability by keeping an adequate management of inbreeding. There exist different methods to manage the inbreeding levels within a population based on pedigree or genomic information (Wang, 2016). A method that has been traditionally used is to avoid matings of related animals tracing the pedigree back for some generations (Weigel and Lin, 2000). There are also more complex methods that involve working at population or animal level, for example, minimizing the average coancestry between mating pairs (Fernández et al., 2000) or optimal contribution selection and maximizing genetic response while constraining progeny inbreeding (Wray and Goddard, 1994; Meuwissen, 1997; Grundy et al., 1998; Howard et al., 2017). However, many factors other than inbreeding play an important role in the effectiveness and the applicability of these strategies. Within these factors, there is the preference of the breeder when choosing a sire and replacement animals within a herd and economical constraints, among others. Consequently, it is complicated to develop tools for sire selection or mating allocation that take into account all the factors involved (Meuwissen, 1997). Considering the period 1999 to 2019 , the increase in pedigree-based inbreeding was $2.67 \%$. Using our inbreeding depression estimates (per 1\% increase 
in inbreeding), the reduction due to inbreeding was $-0.091 \mathrm{~kg}$, $-0.740 \mathrm{~kg},-2.149 \mathrm{~kg}$, and $-0.062 \mathrm{~cm}$ for BW, WW, FW, and SC, respectively, in this period. In turn, the genetic gain in the same period (computed as the difference in the adjusted means of EBV) was $0.005 \mathrm{~kg}, 6.033 \mathrm{~kg}, 12.666 \mathrm{~kg}$, and $0.567 \mathrm{~cm}$ for BW, WW, FW, and SC, respectively. Currently, the increase in inbreeding and the observed inbreeding depression have not yet offset the genetic gain.

Among the various methods that estimate genomic inbreeding, those based on $\mathrm{ROH}$ are currently the most popular (Baes et al., 2019; Maltecca et al., 2020; Sumreddee et al., 2020; Antonios et al., 2021). Several criteria are needed for identifying $\mathrm{ROH}$. The minimum length of the segment, the minimum number of markers, the maximum distance allowed between two consecutive homozygous markers, and the number of missing genotypes allowed must be defined previously to the analysis of each dataset, being highly sensible to the genotyping technology used. This approach is rule based and somewhat arbitrary. Indeed, the criteria for identification and characterization of $\mathrm{ROH}$ are highly variable among and within species, which produces high variability in estimations of inbreeding as reported by Peripolli et al. (2017). The underlying HMM approach, proposed by Druet and Gautier (2017) and implemented in the program $\mathrm{ZooRoH}$, does not require this prior information to decompose individual genomes as mosaic of HBD and non-HBD classes, because it fits individual genetic data with a full probabilistic modeling of the identity-by-descent process along the chromosomes. Moreover, previous HHM approaches like the one of Leutenegger et al. (2003) considered only two states (HBD or non-HBD) when classifying chromosome segments, with no intermediate values. This implies that all the individual inbreeding comes from one or several ancestors from a single generation in the past, or equivalently, that all the HBD segments have the same expected length. Such assumption may be unrealistic in livestock populations such as the Argentinean Brangus, with a complex demographic history with multiple sources of individual inbreeding. We were able to capture more than two classes of inbreeding from different ancestral origins with the approach of Druet and Gautier (2017). We could distinguish 1) a peak of inbreeding between 8 and 16 generations ago, consistent with the bottlenecks due to breed formation in Argentina, 2) a similar contribution across animals of ancient inbreeding (captured by the older HBD class), and 3) variation in the contribution of recent inbreeding classes associated with a strong artificial selection, which was evidenced by a reduction of the effective number of bull sires across generations since breed formation (Cantet, 2013).

\section{Conclusions}

Inbreeding depression was observed for growth traits and SC in Argentinean Brangus cattle using both ABLUP and HBLUP models. For FW, inbreeding depression was also confirmed with the use of marker information even for a small genotyped dataset. Genomic information used alone (HMM model-based approach) or combined with pedigree (single-step approach) provides powerful tools to capture and analyze inbreeding depression. Future work could focus on distinguishing between recent and old inbreeding and the fine mapping of which genome segments participate in inbreeding depression, using a larger dataset of genotyped animals. Even if genotyping has a cost, the financial cost of losses due to inbreeding on production traits should be considered in breeding programs.

\section{Supplementary Data}

Supplementary data are available at Journal of Animal Science online.

\section{Acknowledgments}

This study was partially funded by the projects INRASELGEN EpiNet, France; UBACyTs 20020170200413BA and 20020190100252BA from Universidad de Buenos Aires, Argentina; and PICT 2018 1-188-3387 from Agencia Nacional de Ciencia y Tecnología, Argentina. We would especially like to acknowledge and thank the Argentinean Brangus Breeders Association for allowing us to use its genomic, genealogical, and phenotypic databases for the current research.

\section{Conflict of interest statement}

The authors declare no conflicts of interest.

\section{Literature Cited}

Aguilar, I., and I. Misztal. 2008. Recursive algorithm for inbreeding coefficients assuming nonzero inbreeding of unknown parents. J. Dairy Sci. 91(4):1669-1672. doi:10.3168/ jds.2007-0575

Aguilar, I., I. Misztal, S. Tsuruta, A. Legarra, and H. Wang. 2014. PREGSF90-POSTGSF90: computational tools for the implementation of single-step genomic selection and genome-wide association with ungenotyped individuals in BLUPF90 programs. In: International Committee for World Congresses on Genetics Applied to Livestock Production, editor. Proceedings of the 10th World Congress on Genetics Applied to Livestock Production; August 17 to 22, 2014; Vancouver, BC, Canada. Champaign, IL: American Society of Animal Science; p. 680.

Antonios, S., S. T. Rodríguez-Ramilo, I. Aguilar, J. M. Astruc, A. Legarra, and Z. G. Vitezica. 2021. Genomic and pedigree estimation of inbreeding depression for semen traits in the Basco-Béarnaise dairy sheep breed. J. Dairy Sci. 104:3221-3230. doi:10.3168/jds.2020-18761

Baes, C. F., B. O. Makanjuola, F. Miglior, G. Marras, J. T. Howard, A. Fleming, and C. Maltecca. 2019. Symposium Review: The genomic architecture of inbreeding: how homozygosity affects health and performance. J. Dairy Sci. 102:2807-2817. doi:10.3168/jds.2018-15520

Brinks, J. S., R. T. Clark, and N. M. Kieffer. 1965. Evaluation of response to selection and inbreeding in a closed line of Hereford cattle. Washington, DC: USDA Tech. Bull. No. 1323.

Burrow, H. M. 1993. The effects of inbreeding in beef cattle. Anim. Breed. Abstr. 61(11):737-751. https://www.researchgate.net/ profile/H-Burrow/publication/285667729_The_effects_of inbreeding_in_beef_cattle/links/58c9b040aca27286b3af9f32/ The-effects-of-inbreeding-in-beef-cattle.pdf

Burrow, H. M. 1998. The effects of inbreeding on productive and adaptive traits and temperament of tropical beef cattle. Livest. Prod. Sci. 55(3):227-243. doi:10.1016/ S0301-6226(98)00139-0

Cantet, R. J. C. 2013. La otra carne de la historia: mejoramiento genético en Brangus y Braford en Argentina. Anales Acad. Nac. Agron. Veterin. LXVII:139-160. Argentina. https://1library.co/ document/1y9d7drq-anales-tomo-lxvii.html

Carrillo, J. A., and F. Siewerdt. 2010. Consequences of longterm inbreeding accumulation on preweaning traits in a closed nucleus Angus herd. J. Anim. Sci. 88:87-95. doi:10.2527/ jas.2009-1897

Charlesworth, D., and J. H. Willis. 2009. The genetics of inbreeding depression. Nat. Rev. Genet. 10:783-796. doi:10.1038/nrg2664 
Chen, C., J. P. Steibel, and R. J. Tempelman. 2017. Genomewide association analyses based on broadly different specifications for prior distributions, genomic windows, and estimation methods. Genetics 206:1791-1806. doi:10.1534/ genetics.117.202259

Christensen, O. F., and M. S. Lund. 2010. Genomic prediction when some animals are not genotyped. Genet. Sel. Evol. 42:2. doi:10.1186/1297-9686-42-2

Curik, I., M. Ferenčaković, and J. Sölkner. 2014. Inbreeding and runs of homozygosity: a possible solution to an old problem. Livest. Sci. 166:26-34. doi:10.1016/j.livsci.2014.05.034

Davis, M. E., and R. C. Simmen. 2010. Estimates of inbreeding depression for serum insulin-like growth factor I concentrations, body weights, and body weight gains in Angus beef cattle divergently selected for serum insulinlike growth factor I concentration. J. Anim. Sci. 88:552-561. doi:10.2527/jas.2009-2232

Druet, T., and M. Gautier. 2017. A model-based approach to characterize individual inbreeding at both global and local genomic scales. Mol. Ecol. 26(20):5820-5841. doi:10.1111/ mec.14324

Falcão, A. J. da S., R. Martins Filho, C. de U. Magnabosco, R. Bozzi, and F. de A. M. Lima. 2001. Effects of inbreeding on reproductive and growth traits, and breeding values in a closed Brown Swiss herd. Rev. Bras. Zootec. 30(1):83-92. doi:10.1590/S1516-35982001000100014

Falconer, D. S., and T. F. C. Mackay. 1996. Introduction to quantitative genetics. Edinburgh, UK: Pearson Education Limited.

FAO. 2013. In vivo conservation of animal genetic resources. FAO animal production and health guidelines. No. 14. Rome: FAO.

Fernández, B., E. Santiago, M. A. Toro, and A. Caballero. 2000. Effect of linkage on the control of inbreeding in selection programmes. Genet. Sel. Evol. 32:249-264. doi:10.1186/1297-9686-32-3-249

Garcia-Baccino, C. A., A. Legarra, O. F. Christensen, I. Misztal, I. Pocrnic, Z. G. Vitezica, and R. J. C. Cantet. 2017. Metafounders are related to $\mathrm{F}_{\mathrm{st}}$ fixation indices and reduce bias in singlestep genomic evaluations. Gen. Sel. Evol. 49(1):34. doi:0.1186/ s12711-017-0309-2

Garcia-Baccino, C. A., D. A. L. Lourenco, S. Miller, R. J. C. Cantet, and Z. G. Vitezica, 2020. Estimating dominance genetic variances for growth traits in American Angus males using genomic models. J. Anim. Sci. 98(1):1-7. doi:10.1093/jas/skz384

Goszczynski, D. E., C. M. Corbi-Botto, H. M. Durand, A. RogbergMuñoz, S. Munilla, P. Peral-Garcia, R. J. C. Cantet, and G. Giovambattista. 2018. Evidence of positive selection towards Zebuine haplotypes in the BoLA region of Brangus cattle. Animal 12:215-223. doi:10.1017/S1751731117001380

Grundy, B., B. Villanueva, and J. A. Woolliams. 1998. Dynamic selection procedures for constrained inbreeding and their consequences for pedigree development. Genet. Res. 72(2):159168. doi:10.1017/S0016672398003474

Hill, W. G., and B. S. Weir. 2011. Variation in actual relationship as a consequence of Mendelian sampling and linkage. Genet. Res. (Camb). 93:47-64. doi:10.1017/S0016672310000480

Howard, J. T., J. E. Pryce, C. Baes, and C. Maltecca. 2017. Invited Review: Inbreedingin the genomics era: inbreeding, inbreeding depression, and management of genomic variability. J. Dairy Sci. 100:6009-6024. doi:10.3168/jds.2017-12787

Jacquard, A. 1974. Genetic relationships between relatives. In: Krickeberg, K., R. C. Lewontin, J. Neyman, and M. Schreiber, editors. The genetic structure of populations. Biomathematics. Vol 5. Berlin, Heidelberg: Springer-Verlag; pp. 102-140. doi:10.1007/978-3-642-88415-3_7

Legarra, A., I. Aguilar, and J. J. Colleau. 2020. Short Communication: Methods to compute genomic inbreeding for ungenotyped individuals. J. Dairy Sci. 103(4):3363-3367. doi:10.3168/jds.2019-17750

Legarra, A., I. Aguilar, and I. Misztal. 2009. A relationship matrix including full pedigree and genomic information. J. Dairy Sci. 92:4656-4663. doi:10.3168/jds.2009-2061
Legarra, A., A. Ricard, and L. Varona. 2018. GWAS by GBLUP: single and multimarker EMMAX and Bayes factors, with an example in detection of a major gene for horse gait. G3 (Bethesda). 8:2301-2308. doi:10.1534/g3.118.200336

Leroy, G. 2014. Inbreeding depression in livestock species: review and meta-analysis. Anim. Genet. 45:618-628. doi:10.1111/ age.12178

Leutenegger, A. L., B. Prum, E. Génin, C. Verny, A. Lemainque, F. Clerget-Darpoux, and E. A. Thompson. 2003. Estimation of the inbreeding coefficient through use of genomic data. Am. J. Hum. Genet. 73:516-523. doi:10.1086/378207

Malécot, G. 1948. Les mathématiques de l'hérédité. Paris: Masson et Cie.

Maltecca, C., F. Tiezzi, J. B. Cole, and C. Baes. 2020. Symposium Review: Exploiting homozygosity in the era of genomicsselection, inbreeding, and mating programs. J. Dairy Sci. 103:5302-5313. doi:10.3168/jds.2019-17846

McQuillan, R., A. L. Leutenegger, R. Abdel-Rahman, C. S. Franklin, M. Pericic, L. Barac-Lauc, N. Smolej-Narancic, B. Janicijevic, O. Polasek, A. Tenesa, et al. 2008. Runs of homozygosity in European populations. Am. J. Hum. Genet. 83:359-372. doi:10.1016/j.ajhg.2008.08.007

Meuwissen, T. H. 1997. Maximizing the response of selection with a predefined rate of inbreeding. J. Anim. Sci. 75:934-940. doi:10.2527/1997.754934x

Misztal, I., S. Tsuruta, T. Strabel, B. Auvray, T. Druet, and D. Lee. 2002. BLUPF90 and related programs (BGF90). In: World Congress on Genetics Applied to Livestock Production Organizing Committee, editor. Proceedings of the 7th World Congress on Genetics Applied to Livestock Production. Comm. No. 28.07. August 19-23, 2002; Montpellier, France; France: INRA, Castanet-Tolosan.

Paim, T. D. P., E. H. A. Hay, C. Wilson, M. G. Thomas, L. A. Kuehn, S. R. Paiva, C. McManus, and H. Blackburn. 2020. Genomic breed composition of selection signatures in Brangus beef cattle. Front. Genet. 11:710. doi:10.3389/ fgene.2020.00710

Pereira, R. J., M. L. Santana, Jr, D. R. Ayres, A. B. Bignardi, G. R. Menezes, L. O. Silva, C. H. Machado, L. A. Josahkian, and L. G. Albuquerque. 2016. Inbreeding depression in Zebu cattle traits. J. Anim. Breed. Genet. 133:523-533. doi:10.1111/ jbg.12219

Peripolli, E., D. P. Munari, M. V. G. B. Silva, A. L. F. Lima, R. Irgang, and F. Baldi. 2017. Runs of homozygosity: current knowledge and applications in livestock. Anim. Genet. 48:255-271. doi:10.1111/age.12526

Ron Garrido, L. J., A. N. Birchmeier, S. Munilla, and R. J. C. Cantet. 2008. Estimation of effective population size using bivariate discrete distributions for modeling family size in beef cattle. Livest. Sci. 117:43-51. doi:10.1016/j. livsci.2007.11.008

Santana, M. L. Jr, P. S. Oliveira, V. B. Pedrosa, J. P. Eler, E. Groeneveld, and J. B. S. Ferraz. 2010. Effect of inbreeding on growth and reproductive traits of Nellore cattle in Brazil. Livest. Sci. 131:212-217. doi:10.1016/j.livsci.2010.04.003

Sumreddee, P., S. Toghiani, E. H. Hay, A. Roberts, S. E. Agrrey, and R. Rekaya. 2018. Inbreeding depression in line 1 Hereford cattle population using pedigree and genomic information. J. Anim. Sci. 97(1):1-18. doi:10.1093/jas/sky385

Sumreddee, P., S. Toghiani, E. H. Hay, A. Roberts, S. E. Aggrey, and R. Rekaya. 2020. Runs of homozygosity and analysis of inbreeding depression. J. Anim. Sci. 98(12):1-11. doi:10.1093/ jas/skaa361

Swiger, L. A., K. E. Gregory, R. M. Koch, and V. A. Arthaud. 1961. Effect of inbreeding on performance traits of beef cattle. J. Anim. Sci. 20(3):626-630. doi:10.2527/jas1961.203626x

VanRaden,P.M.1992.Accounting forinbreedingand crossbreeding in genetic evaluation of large populations. J. Dairy Sci. 75(11):3136-3144. doi:10.3168/jds.S0022-0302(92)78077-1

VanRaden, P. M. 2008. Efficient methods to compute genomic predictions. J. Dairy Sci. 91:4414-4423. doi:10.3168/ jds.2007-0980 
Villanueva, B., A. Fernández, M. Saura, A. Caballero, J. Fernández, E. Morales-González, M. A. Toro, and R. Pong-Wong. 2021. The value of genomic relationship matrices to estimate levels of inbreeding. Genet. Sel. Evol. 53:42. doi:10.1186/s12711-021-00635-0

Wang, J. 2016. Pedigrees or markers: which are better in estimating relatedness and inbreeding coefficient? Theor. Popul. Biol. 107:4-13. doi:10.1016/j.tpb.2015.08.006
Weigel, K. A., and S. W. Lin. 2000. Use of computerized mate selection programs to control inbreeding of Holstein and Jersey cattle in the next generation. J. Dairy Sci. 83(4):822-828. doi:10.3168/jds.S0022-0302(00)74945-9

Wray, N. R., and M. E. Goddard. 1994. Increasing long-term response to selection. Genet. Sel. Evol. 26(5):431-451. doi:10.1186/1297-9686-26-5-431 